\title{
Parallelization of All-Pairs-Shortest-Path Algorithms in Unweighted Graph
}

\author{
Masahiro Nakao \\ RIKEN Center for Computational \\ Science \\ Hyogo, Japan
}

\author{
Hitoshi Murai \\ RIKEN Center for Computational \\ Science \\ Hyogo, Japan
}

\author{
Mitsuhisa Sato \\ RIKEN Center for Computational \\ Science \\ Hyogo, Japan
}

\begin{abstract}
The design of the network topology of a large-scale parallel computer system can be represented as an order/degree problem in graph theory. To solve the order/degree problem, it is necessary to obtain an all-pairs-shortest-path (APSP) of the graph. Thus, this paper evaluates two parallel algorithms that quickly find the APSP in unweighted graphs and compares their performance. The first APSP algorithm is based on the breadth-first search (BFS-APSP) and the second is based on the adjacency matrix (ADJ-APSP). First, we develop serial algorithms and threaded algorithms using OpenMP, and show that ADJ-APSP is up to 32.34 times faster than BFSAPSP. Next, we develop hybrid-parallel algorithms using OpenMP and MPI, and show that BFS-APSP is faster than ADJ-APSP under certain conditions because the maximum number of processes in BFS-APSP is greater than in ADJ-APSP. In addition, we parallelize ADJ-APSP using a single GPU (NVIDIA Tesla V100) and achieve a speed increase of up to 16.53-fold compared to that of a single CPU. Finally, we evaluate the performance of the algorithms using 128 GPUs and achieve a computation time 101.10 times faster than that using a single GPU. Moreover, it is shown that the calculation time of both algorithms can be greatly reduced when the input graphs are symmetric.
\end{abstract}

\section{CCS CONCEPTS}

- Theory of computation $\rightarrow$ Massively parallel algorithms.

\section{KEYWORDS}

hybrid parallelization, GPU, network, graph theory

\section{ACM Reference Format:}

Masahiro Nakao, Hitoshi Murai, and Mitsuhisa Sato. 2020. Parallelization of All-Pairs-Shortest-Path Algorithms in Unweighted Graph. In International Conference on High Performance Computing in Asia-Pacific Region (HPCAsia2020), fanuary 15-17, 2020, Fukuoka, Japan. ACM, New York, NY, USA, 10 pages. https://doi.org/10.1145/3368474.3368478

\section{INTRODUCTION}

In large-scale parallel computer systems such as supercomputers and data centers, many calculation nodes are interconnected via

Permission to make digital or hard copies of all or part of this work for personal or classroom use is granted without fee provided that copies are not made or distributed for profit or commercial advantage and that copies bear this notice and the full citation on the first page. Copyrights for components of this work owned by others than ACM must be honored. Abstracting with credit is permitted. To copy otherwise, or republish, to post on servers or to redistribute to lists, requires prior specific permission and/or a fee. Request permissions from permissions@acm.org.

HPCAsia2020, January 15-17, 2020, Fukuoka, Japan

(C) 2020 Association for Computing Machinery.

ACM ISBN 978-1-4503-7236-7/20/01 \$ \$15.00

https://doi.org/10.1145/3368474.3368478 a network. To achieve optimal performance on these systems, it is important to design the network topology so that the diameter and average distance of the number of hops between calculation nodes are small[2, 12, 14, 23]. To obtain small diameters and average distances, it is necessary to calculate an all-pairs-shortest-path (APSP) between the calculation nodes.

By considering the calculation node as "vertex" and the network wiring as "edge", the network topology can be represented as a graph. Thus, designing a network topology with small diameter and average distance can be defined as an order/degree problem in graph theory[15]. The order/degree problem consists of finding the graph with the smallest diameter and average distance from a set of graphs with the given number of vertices (order) and degree. In this paper, the order/degree problem assumes that the performance of the network between calculation nodes is the same, so it uses unweighted graphs. The order/degree problem can be applied not only to networks in parallel computer systems but also to networks in various industrial products such as Network on a Chip[11].

Although the definition of the order/degree problem is simple, no efficient method for solving it has thus far been found. For this reason, metaheuristic algorithms such as simulated annealing $(\mathrm{SA})[7,9]$ are often used[13, 16, 17]. However, metaheuristic algorithms require many calculations to obtain the solution for the current state of a system. In the order/degree problem, the diameter and the average distance are often used as the evaluation criteria. The computational complexity of the APSP algorithm based on the breadth-first search (BFS-APSP) is $O\left(n^{2} d\right)$ for $n$ vertices and degree $d[13]$. Therefore, as the number of vertices increases, the computation time for APSP increases quickly.

In our previous research[13], we reduced the computation time for APSP by parallelizing BFS-APSP using MPI and OpenMP. In this paper, the APSP algorithm based on the adjacency matrix (ADJAPSP) is parallelized and compared with BFS-APSP. Furthermore, we also develop a GPU version of ADJ-APSP and evaluate the performance. The previous research also indicated that the calculation time using BFS-APSP can be drastically reduced and the solution search performance of SA improved by using a graph symmetry. Therefore, this paper also uses the graph symmetry in ADJ-APSP to reduce the calculation time. All the programs used in this paper can be obtained at https:/github.com/mnakao/APSP/.

The remainder of this paper is structured as follows. Section 2 gives an overview of the order/degree problem and reviews existing APSP algorithms. Section 3 describes the parallelization of BFSAPSP and ADJ-APSP. Section 4 evaluates and compares the algorithms. Section 5 describes the parallelization of ADJ-APSP using GPU and the graph symmetry for each APSP algorithm. Section 6 summarizes the results and suggests areas for future research. 

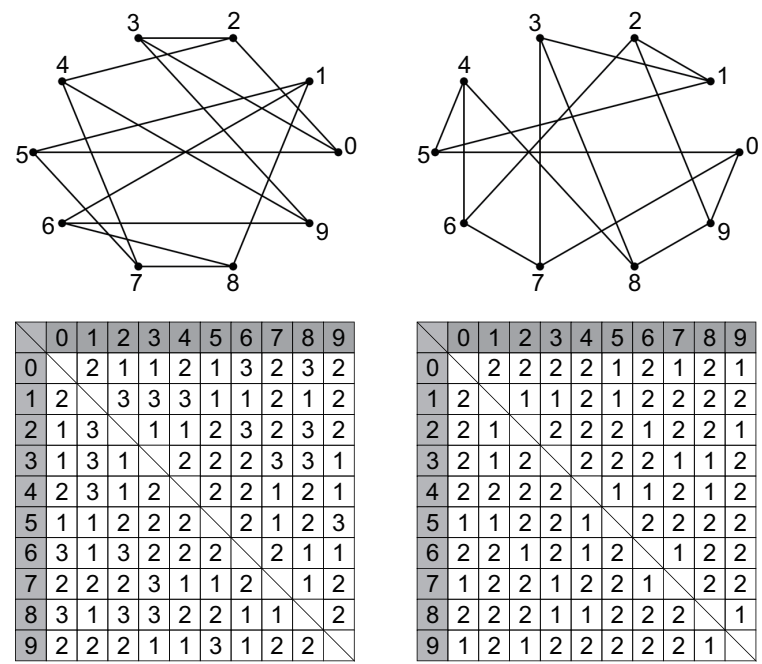

(a) Dia.=3, Ave. Dist. $=1.89$

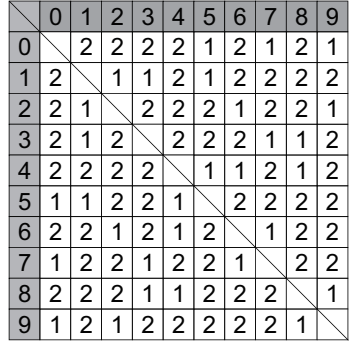

(b) Dia.=2, Ave. Dist. $=1.67$

Figure 1: Example of Graphs $(n, d)=(10,3)[13]$

\section{RELATED WORK}

\subsection{Order/degree problem}

The aim of the order/degree problem is to minimize the diameter and average distance of a graph with $n$ vertices and degree $d$. Fig. 1 shows an example of graphs with $(n, d)=(10,3)$ and their distance matrixes. The distance matrix represents the distance between the vertices of the graph. Since both graphs shown in Fig. 1 are undirected graphs, the distance matrices are symmetric. While the diameter is the maximum value of the elements in the distance matrix, the average distance is the total for all elements in the distance matrix divided by the number of elements $\left(n^{2}-n\right)$. Because Fig. $1 \mathrm{~b}$ has a smaller diameter and average distance, it can be said that Fig. $1 \mathrm{~b}$ is a more optimal solution to the order/degree problem than Fig. 1a.

An international competition for finding new algorithms for the order/degree problem, called "Graph Golf[16]", has been held by the National Institute of Informatics since 2015. Graph Golf provides problems containing graphs with different combinations of vertices and degrees every year. The problems in 2019 are $(n, d)=$ $(50,4),(512,4),(512,6),(1024,4),(1726,30),(4855,15),(9344,6)$, $(65536,6),(100000,8),(1000000,16),(1000000,32)$. Note that the number of vertices is fixed, but the value of the degree given is the maximum allowed value. For example, $(4855,15)$ has an odd number of vertices and degree, so there is no graph with 15 edges on all vertices due to the handshaking lemma. Participants of Graph Golf can post their own graphs on the official website within the competition period (May 13 to October 14, 2019).

The official website publishes some slides and papers on methods developed by past participants[17, 19, 24]. The methods can be broadly divided into two groups. (1) Metaheuristic algorithm such as SA. While this method has the advantage of creating graphs with an arbitrary number of vertices and degree, it has very high computational costs for APSP. To overcome this problem, approximations may be used to calculate APSP. However, the approximation error may adversely affect the solution search performance of metaheuristic algorithms. Therefore, we consider that it is important to obtain APSP accurately. (2) Combine multiple graphs with a small number of vertices. While this method has the advantage that the number of APSP calculations is smaller than (1), only graphs with a specific number of vertices and degree can be created. In addition, when creating a graph with a large number of vertices, the number of possible combinations becomes enormous, so it is also important to reduce the APSP calculation time in (2).

Another problem similar to order/degree problem is a degree/diameter problem[1]. This is an important problem in graph theory that finds a graph with as many vertices as possible while satisfying the given degree and diameter. This paper is beneficial because similar calculations are required for the degree/diameter problem.

\subsection{All-pairs-shortest-path algorithm}

Since APSP is one of the most important features of a weighted graph, various APSP algorithms in weighted graphs have been proposed. By assuming that the graph's weights are uniform, the APSP algorithms in weighted graphs can be used for unweighted graphs, but the computational efficiency may be low. For example, the computational complexity of the Warshall-Floyd algorithm [18, 22], which is the most famous APSP algorithm in weighted graphs, is $\mathrm{O}\left(n^{3}\right)$. On the other hand, the computational complexity of BFS-APSP is $\mathrm{O}\left(n^{2} d\right)$. Since the graph in the order/degree problem is a simple graph containing no loops or multiple edges, $n>d$ always holds. That is, since $\mathrm{O}\left(n^{3}\right)>\mathrm{O}\left(n^{2} d\right)$, BFS-APSP is more computationally efficient than the Warshall-Floyd algorithm.

As far as the authors know, no research on the parallelization of APSP algorithms in unweighted graphs has been conducted. As an efficient serial APSP algorithm in unweighted undirected graphs, the Seidel algorithm was proposed[21], which has a computational complexity of $\mathrm{O}\left(n^{2.376} \log n\right)$. That is, if $n^{0.376} \log n<d$ (in the case of a dense graph with a relatively large number of edges relative to the number of vertices), the Seidel algorithm is more computationally efficient than BFS-APSP. However, the order/degree problem is characterized by its relatively low degree because it is assumed it can be used for industrial products. For example, all the Graph Golf 2019 problems described in section 2.1 are $n^{0.376} \log n>d$. Therefore, the Seidel algorithm is not treated in this paper.

\section{PARALLELIZATION OF ALL-PAIRS-SHORTEST-PATH ALGORITHM}

This section explains the serial BFS-APSP and ADJ-APSP algorithms, and then reviews parallel algorithms using MPI and OpenMP.

\subsection{Algorithms by breadth-first search}

3.1.1 Overview. BFS can be used to find the distances from one vertex to others. Therefore, APSP can be obtained by BFS for all vertices. The computational complexity of applying BFS to one vertex is proportional to the number of edges; $O(n d)$. When it is repeated $n$ times, the computational complexity of BFS-APSP is $O\left(n^{2} d\right)$. 


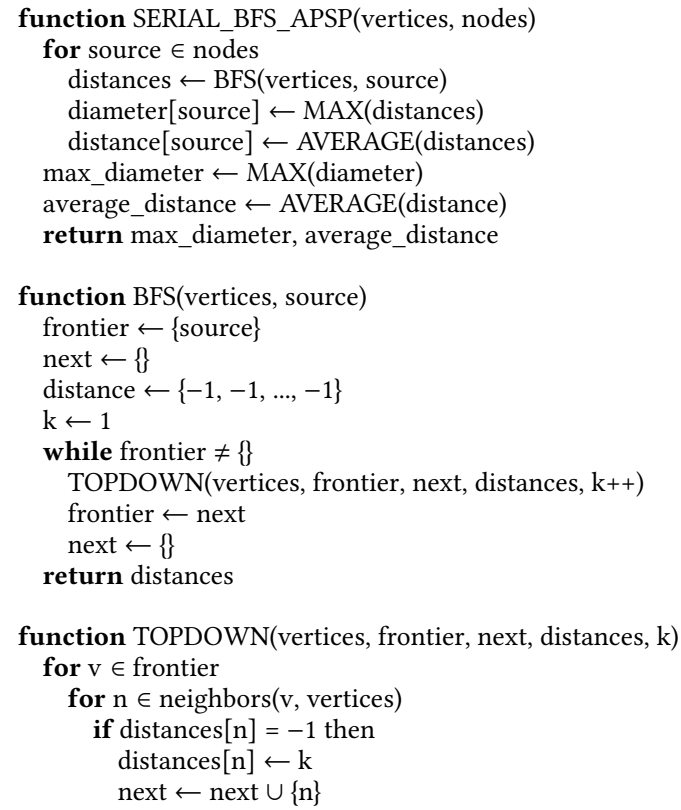

\section{Figure 2: Serial BFS-APSP}

3.1.2 Serial algorithm. Fig. 2 shows the pseudo code for the serial BFS-APSP. We adopt the top-down approach of the level synchronized BFS[8]. In lines $2-5$, the loop statement shows that the BFS starting from a certain vertex is repeated as many times as the number of vertices. In lines 6-7, the diameter and average distance of the graph are calculated from the distance information obtained by BFS. The functions BFS() and TOPDOWN() are almost the same as in [8], so the explanation is omitted. Although omitted in Fig. 2, the optimization by bitmap[10] is also performed to increase the cache hit rate.

As an extension to the above approach, [8] proposes a hybrid approach for Kronecker graphs[5], which have patterns that often appear in social networks. In a Kronecker graph, vertices with a large degree and vertices with a low degree are mixed. In such a graph, the hybrid approach can perform BFS faster than the topdown approach. However, the graphs for the order/degree problem have almost the same degree, and their degree is relatively small. As a result of preliminary experiments, the top-down approach was superior to the hybrid approach, so we select the top-down approach in this paper.

3.1.3 Parallel algorithm using MPI and OpenMP. To overview the effect of parallelization, multiple BFSs are performed simultaneously using MPI, and one BFS is divided into threads using OpenMP. Thus, the maximum number of processes is $n$.

Fig. 3 shows the pseudo code of the parallel BFS-APSP. In lines $2-5$, starting points are assigned to each process evenly, and BFSs are executed in parallel. In lines $6-8$, the diameter and average distance of the graph are calculated by reducing the distance information on each process using collective communication. The algorithm BFS () is the same as that in Fig. 2. In lines 24-35, each thread searches the vertex set frontier separately. In lines 28-35,

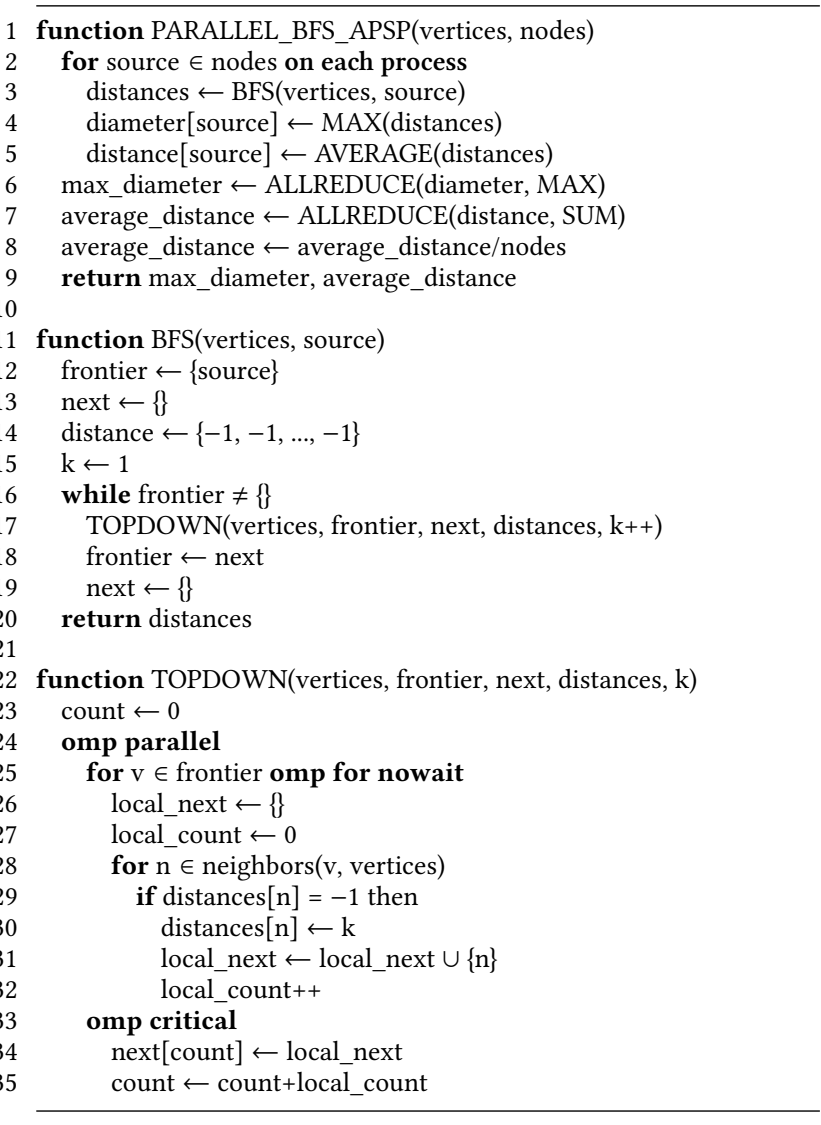

Figure 3: Parallel BFS-APSP

each thread stores the obtained search points in a thread-private variable local_next and updates a thread-shared variable next from local_next using exclusive control between threads.

\subsection{Algorithms by adjacency matrix}

3.2.1 Overview. Let $A$ be an adjacency matrix of the graph. If the value of an element $a_{i, j}$ in $A^{k}$ is 1 , it means that the vertex $i$ can reach the vertex $j$ within $k$ hops. By using this property, the diameter and average distance of the graph can be obtained. As $k$ is increased in increments of 1 , the value of $k$ is the diameter when all elements are 1. Also, every time $k$ is increased from 1 to the diameter, the total distance between vertices can be obtained by summing all the elements whose value for element $a_{i, j}$ in $A^{k}$ is 0 (the unreachable vertices at the $k$ th hop). The average value is the total distance divided by the number of elements $\left(n^{2}-n\right)$.

The computational complexity of ADJ-APSP is $\mathrm{O}\left(n^{2} d D / E\right)$, where the diameter is $D$ and the number of bits in one element of the adjacency matrix is $E$. In general, the value of $D$ of graphs in the order/degree problem is small due to the small-world effect. For example, even for a random graph with vertices and edges connected randomly, the value of $D$ for all the Graph Golf 2019 problems described in section 2.1 is less than 10 . Since our implementation uses the uint64_t in $C$ language for the adjacency matrix, $E=64$. 


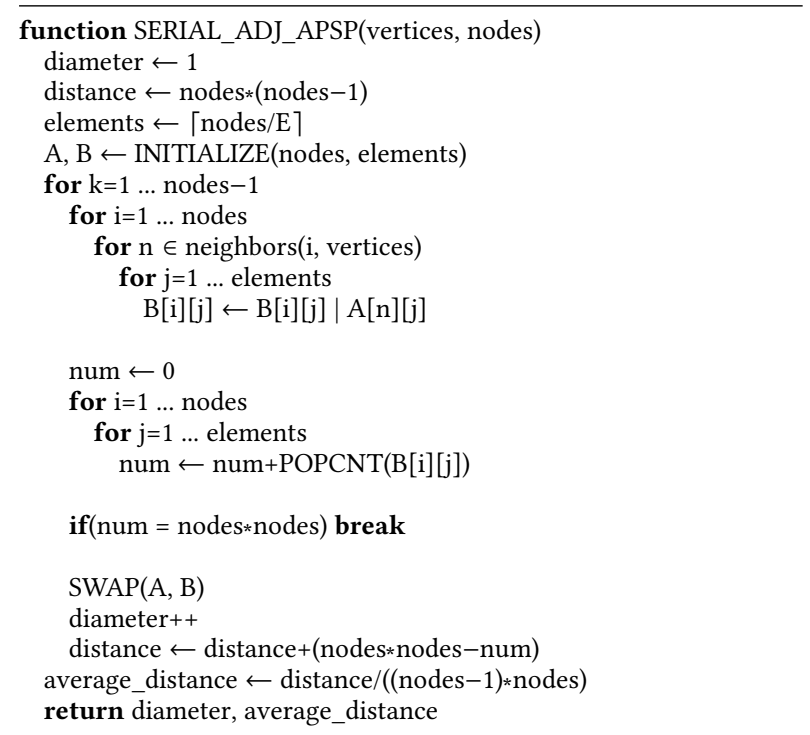

Figure 4: Serial ADJ-APSP

\begin{tabular}{|c|c|c|c|}
\hline A & $\mathrm{nbr}$ & $A^{1}$ & $A^{2}$ \\
\hline 0000000001 & 235 & 0000101101 & 1010111111 \\
\hline 000000010 & 568 & 0101100010 & 1111100011 \\
\hline 0000000100 & 034 & 0000011101 & 1010111101 \\
\hline 0000001000 & 029 & 1000001101 & 1001111101 \\
\hline 0000010000 & 279 & 1010010100 & 1111111101 \\
\hline 0000100000 & 017 & 0010100011 & 0111111111 \\
\hline 0001000000 & 189 & 1101000010 & 1111111010 \\
\hline 0010000000 & 458 & 0110110000 & 1111110111 \\
\hline 0100000000 & 167 & 0111000010 & 1111110010 \\
\hline 1000000000 & 346 & 1001011000 & 1111011111 \\
\hline
\end{tabular}

Figure 5: Example of ADJ-APSP

\begin{tabular}{|c|c|c|c|c|c|}
\hline$A_{1}$ & $\mathrm{nbr}$ & $A_{1}^{1}$ & $A_{0}$ & $\mathrm{nbr}$ & $A_{0}^{1}$ \\
\hline 00000 & 235 & 00001 & 00001 & 235 & 01101 \\
\hline 00000 & 568 & 01011 & 00010 & 568 & 00010 \\
\hline 00000 & 034 & 00000 & 00100 & 034 & 11101 \\
\hline 00000 & 029 & 10000 & 01000 & 029 & 01101 \\
\hline 00000 & 279 & 10100 & 10000 & 279 & 10100 \\
\hline 00001 & 017 & 00101 & 00000 & 017 & 00011 \\
\hline 00010 & 189 & 11010 & 00000 & 189 & 00010 \\
\hline 00100 & 458 & 01101 & 00000 & 458 & 10000 \\
\hline 0 & 167 & 01110 & 00000 & 167 & 00010 \\
\hline 10000 & 346 & 10010 & 00000 & 346 & 11000 \\
\hline
\end{tabular}

Figure 6: Example of Divided ADJ-APSP

3.2.2 Serial algorithm. Fig. 4 shows the pseudo code of the serial ADJ-APSP algorithm, which is referred to as [20]. First, $n \times n$ bit adjacency matrices $A$ and $B$ are prepared. In line 4 , the number of elements of each column in the adjacency matrix elements is calculated. In line 5 , the $n$th element from the right in the $n$th row is set to 1 for $A$ and $B$, and the others are set to 0 . In line $6, k$ is increased in increments of 1 to calculate $A^{k}$. The reason that the maximum value of $k$ is (nodes - 1) is because the diameter of the graph cannot exceed $(n-1)$. In lines $7-10$, to calculate $A^{k}$, the logical sum is performed using an adjacency list neighbors which stores the neighbor information, and the result is substituted into $B$. In lines $12-15$, a variable num, the number of bits of 1 in $B$, is counted to determine the number of vertices reached at the $k$ th hop. In line 21 , the number of bits of 0 in $B$ is calculated by subtracting num from the total number of elements (nodes $\times$ nodes). The function POPCNT () counts the number bits valued 1 contained in a single element. Depending on the architecture, our implementation uses either__builtin_popcountll() or_mm_popcnt_u64(). In line 17, when all elements are 1 (when all vertices have been reached), the flow leaves the loop statement. In line 19, A and B are swapped for use in the next iteration. In lines 20-22, the diameter and average distance of the graph are calculated.

Fig. 5 shows an example of the serial ADJ-APSP using Fig. 1a. For convenience of implementation, the distance matrix of Fig. 1a and the matrix $A^{k}$ of Fig. 5 are horizontally reversed. The matrix $A$ in Fig. 5 represents the state immediately after initialization. The adjacency list $n b r$ which is the same as neighbors used in line 8 of Fig. 4 stores the adjacency relationship between vertices. In Fig. 5, the changes are written in bold red. The changes from $A$ to $A^{1}$ are the parts corresponding to " 1 " in the distance matrix of Fig. 1a. Similarly, those from $A^{1}$ to $A^{2}$ are the parts corresponding to " 2 ". When the process continues, all bits in $A^{3}$ become " 1 ".
Since the size of the adjacency matrix is $\left(n^{2} / 8\right)$ bytes, the amount of memory used increases in proportion to the square of the number of vertices $n$. For example, the maximum number of vertices in the Graph Golf 2019 problems is $n=1,000,000$, and the size of each adjacency matrix is about 116 gigabytes. Therefore, it is difficult to calculate a large-scale graph on a laptop with the ADJAPSP algorithm in Fig. 4. Therefore, we describe a method which divides the adjacency matrix vertically and calculates each graph independently (hereinafter described as Divided ADJ-APSP). Fig. 6 shows an example of the application of the Divided ADJ-APSP to the graph in Fig. 5 where the adjacency matrix $A$ is divided into two. While the upper 5 bits in $A^{k}$ are $A_{1}^{k}$, the lower 5 bits in $A^{k}$ are $A_{0}^{k}$. When the number of partitions is parsize, the size of the adjacency matrix is $\left(n^{2} / 8 /\right.$ parsize $)$ bytes.

Fig. 7 shows the pseudo code of the serial Divided ADJ-APSP. In line 5, the constant CHUNK is the number of elements of a column in the divided adjacency matrix. In line 6 , the following process is repeated parsize times. From line 7 onwards, the description is omitted because it is almost the same as Fig. 4 except that the constant CHUNK is used instead of the variable elements. Moreover, we omit the case where nodes is not divisible by CHUNK. Note that the value of $(C H U N K \times E)$, the number of bits for each column in an adjacency matrix, should be a multiple of 512 and somewhat large in order to make effective use of SIMD and the memory bandwidth in the logical sum operation in line 12 . Thus, our implementation sets $C H U N K=64$.

In a preliminary experiment, in the performance comparison of ADJ-APSP and Divided ADJ-APSP, it is confirmed that ADJ-APSP has a slightly better performance. Therefore, our implementation automatically selects Divided ADJ-APSP only when dealing with large graphs instead of ADJ-APSP. Specifically, Divided ADJ-APSP 


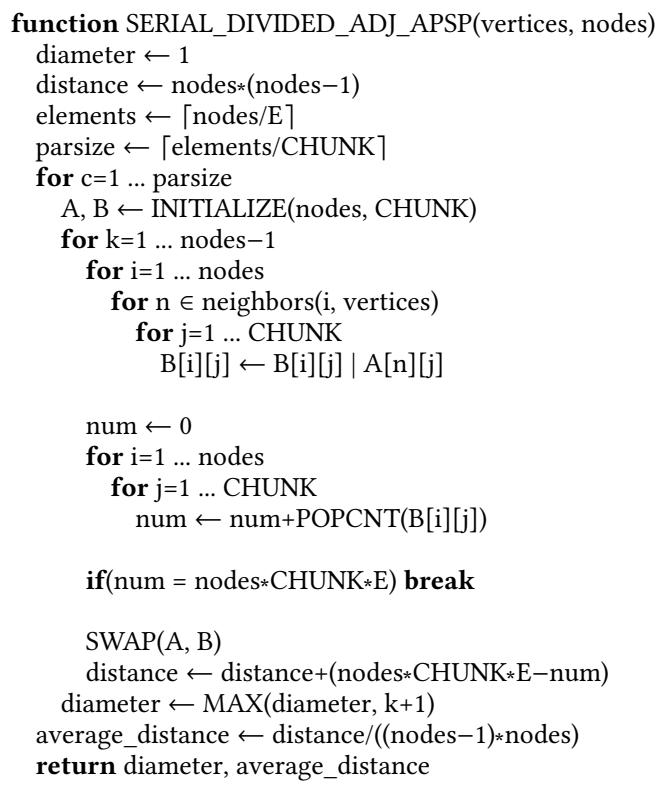

Figure 7: Serial Divided ADJ-APSP

is executed when the size of the adjacency matrix $\left(n^{2} / 8\right)$ exceeds $2^{31}$.

3.2.3 Parallel algorithm using MPI and OpenMP. Using the same division method as Divided ADJ-APSP, it is possible to parallelize ADJ-APSP using MPI. In addition, since some loop statements in ADJ-APSP have no data dependency, thread parallelization using OpenMP can also be performed. The maximum number of processes is $\lceil n / E\rceil$, which is the same as the number of elements in the adjacency matrix.

Fig. 8 shows the pseudo code of the parallel ADJ-APSP. In line 5 , the variable procs is the number of processes, and the variable chunk is the number of elements of a column in the adjacency matrix processed by each process. In line 7 , the iteration of the loop statement is assigned to each process evenly and executed in parallel. In lines 8-24, the variable chunk is used instead of the constant CHUNK in Fig. 7. In lines 10 and 16, OpenMP parallelizes the loop statements. In lines 25-27, each process reduces the distance information by using collective communication to calculate the diameter and average distance of the graph. In line 3, unlike Fig. 7, the initial value of the variable distance is set to 0 . This is because the reducing operation in line 26 is performed on the variable distance. The actual initial value (nodes $*($ nodes -1$)$ ) is equivalent to adding 1 to average_distance in line 27.

Serial Divided ADJ-APSP in Fig. 7 can also be parallelized based on Fig. 8, which is called parallel Divided ADJ-APSP. It can be developed simply by deleting line 5 in Fig. 8 and replacing the variable chunk with the constant CHUNK. As with the serial version, our implementation automatically selects parallel Divided ADJAPSP instead of parallel ADJ-APSP when the adjacency matrix size ( $n^{2} / 8 /$ parsize $)$ exceeds $2^{31}$ to save memory.

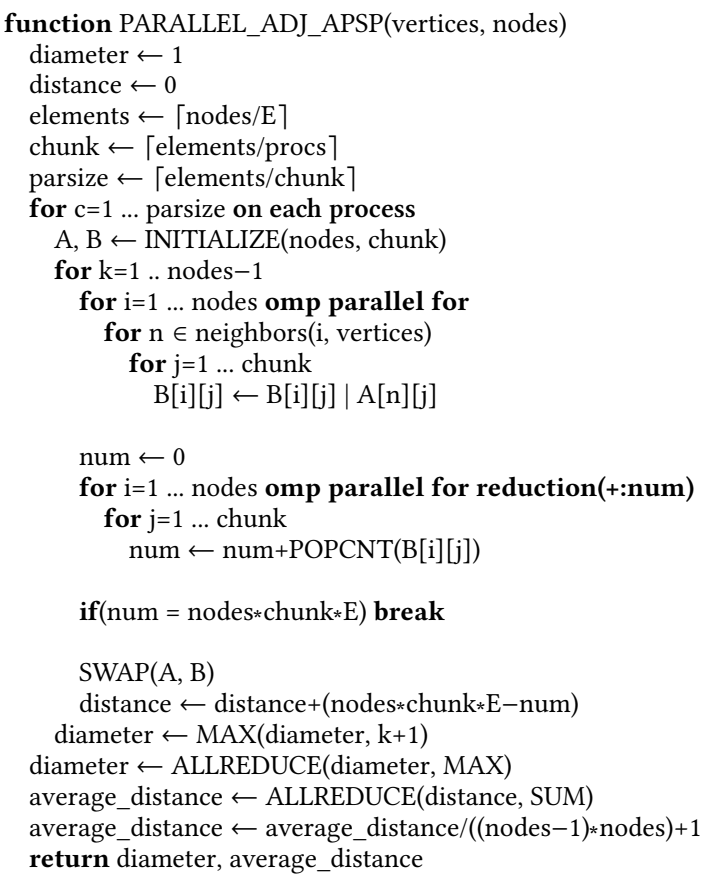

Figure 8: Parallel ADJ-APSP

Table 1: Specification of the $\mathrm{K}$ computer

\begin{tabular}{l|l}
\hline CPU & SPARC64 VIIIfx (8Cores, 2.0GHz) \\
Memory & DDR3 (64GB/s, 16GB) \\
& Torus fusion six-dimensional mesh/torus \\
Network & network, 5GB/s $\times 10$ \\
Software & Fujitsu Compiler K-1.2.0-25 \\
\hline
\end{tabular}

Table 2: Specification of the Cygnus system

\begin{tabular}{l|l}
\hline CPU & Intel Xeon Gold 6126 $(12$ Cores, $2.6 \mathrm{GHz}) \times 2$ \\
Memory & DDR4 $(128 \mathrm{~GB} / \mathrm{s} \times 2,192 \mathrm{~GB})$ \\
GPU & NVIDIA Tesla V100 $(900 \mathrm{~GB} / \mathrm{s}, 32 \mathrm{~GB}) \times 4$ \\
Network & InfiniBand HDR100 $(12.5 \mathrm{~GB} / \mathrm{s}) \times 4$ \\
Software & intel/19.0.3, mvapich/2.3.1, cuda/10.1 \\
\hline
\end{tabular}

\section{EVALUATION}

\subsection{Experiment environment}

This section compares the performance of the two types of APSP algorithms described in section 3. For the experiment, we use the $\mathrm{K}$ computer located in the RIKEN Center for Computational Science and the Cygnus system located in the University of Tsukuba. Table 1 and 2 show the specifications. The graphs used for the evaluations are random graphs provided by the official website of GraphGolf[16].

\subsection{Evaluation of serial algorithm}

This section evaluates the serial algorithms described in sections 3.1.2 and 3.2.2. The graphs used in the evaluation are $(n, d, D)=$ 


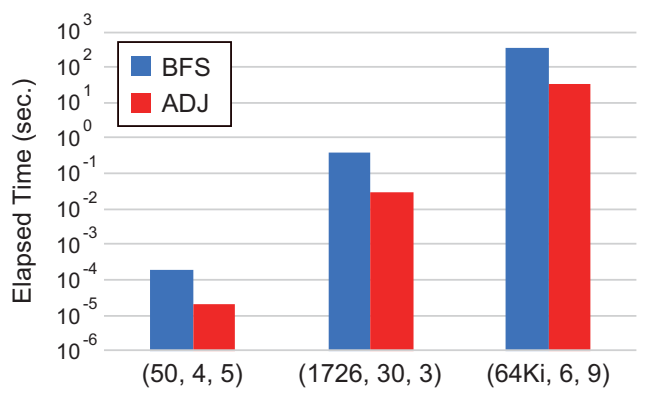

(a) On the K computer

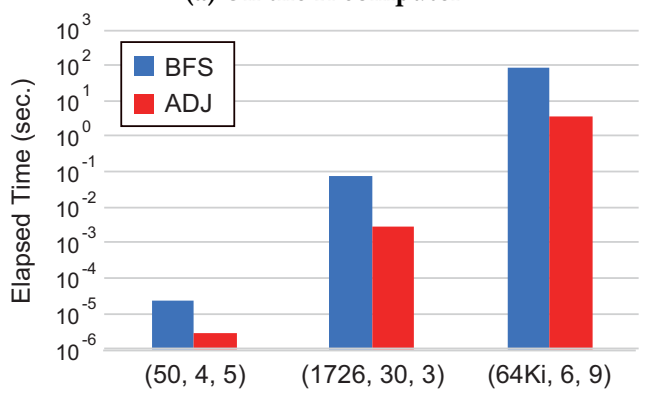

(b) On the Cygnus system

Figure 9: Results of Serial APSP Algorithm

(50, 4, 5), (1726, 30, 3), and (65536, 6, 9). Hereinafter, $(65536,6,9)$ is described as $(64 \mathrm{Ki}, 6,9)$. Note that the APSP algorithm based on the adjacency matrix uses ADJ-APSP instead of Divided ADJ-APSP for all graphs.

From the results shown in Fig. 9, the ADJ-APSP algorithm is faster than the BFS-APSP algorithm for all cases. The computation time is 9.65 to 13.38 times faster on the K computer and 8.08 to 29.49 times faster on the Cygnus system. In addition, the Cygnus system is faster than the K computer, 4.14- to 8.32-fold for BFS-APSP, and 6.96- to 10.40-fold for ADJ-APSP.

\subsection{Evaluation of parallel algorithm}

This section evaluates the parallel algorithms described in sections 3.1.3 and 3.2.3. The graphs used in the evaluation are $(n, d, D)=$ $(64 \mathrm{Ki}, 6,9)$ and $(1000000,32,5)$. Hereinafter, $(1000000,32,5)$ is described as $(1 \mathrm{M}, 32,5)$.

4.3.1 Parallel algorithm by OpenMP. To examine the effects of thread parallelization, the number of processes is fixed at 1 , and the number of threads is changed. However, some results for BFS-APSP in $(1 \mathrm{M}, 32,5)$ could not be obtained because the time limit to complete the execution on the computers was exceeded. Moreover, Divided ADJ-APSP was executed instead of ADJ-APSP in (1M, 32, 5).

Fig. 10 shows the results which indicate that ADJ-APSP is faster than BFS-APSP for the same problem. While the speed difference for the maximum number of threads between ADJ-APSP and BFSAPSP of $(64 \mathrm{Ki}, 6,9)$ on the K computer and the Cygnus system are factors of 19.62 and 32.34, those of $(1 \mathrm{M}, 32,5)$ are 25.27 and 31.29, respectively. Next, Fig. 11 shows the speed increase for Fig. 10. BFSAPSP of $(1 \mathrm{M}, 32,5)$ is not described because it is based on one

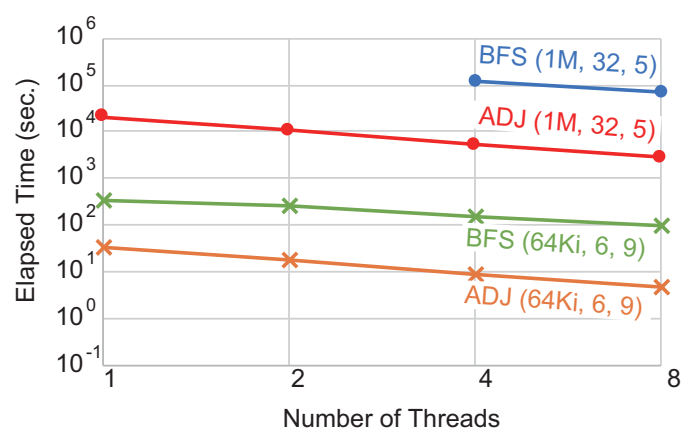

(a) On the K computer

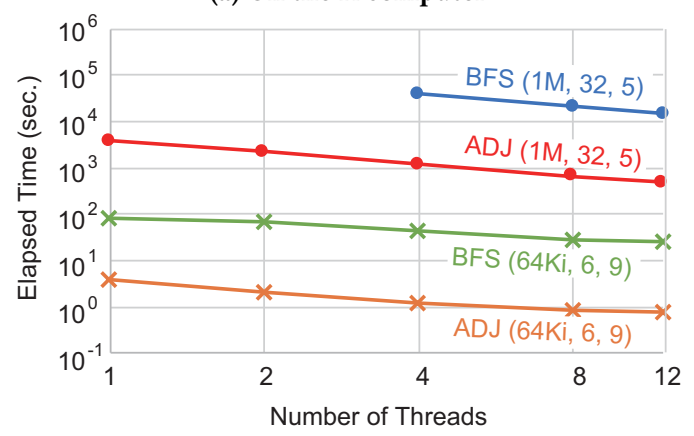

(b) On the Cygnus system

Figure 10: Results of Threaded APSP Algorithm

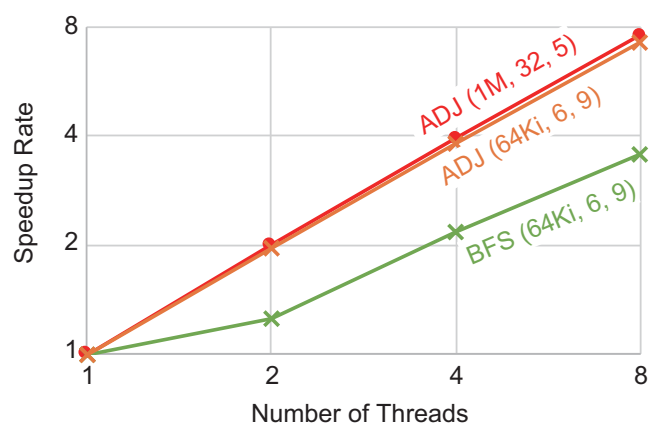

(a) On the K computer

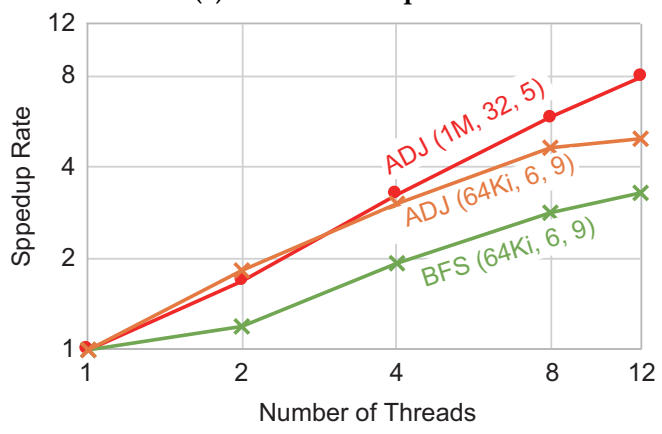

(b) On the Cygnus system

Figure 11: Speedup for Threaded APSP Algorithm 


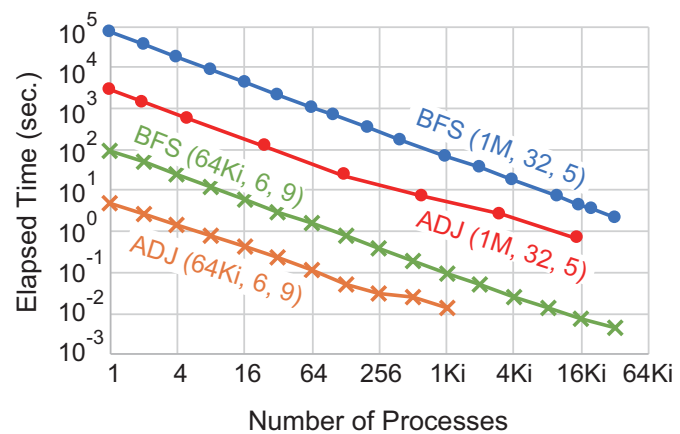

(a) On the K computer

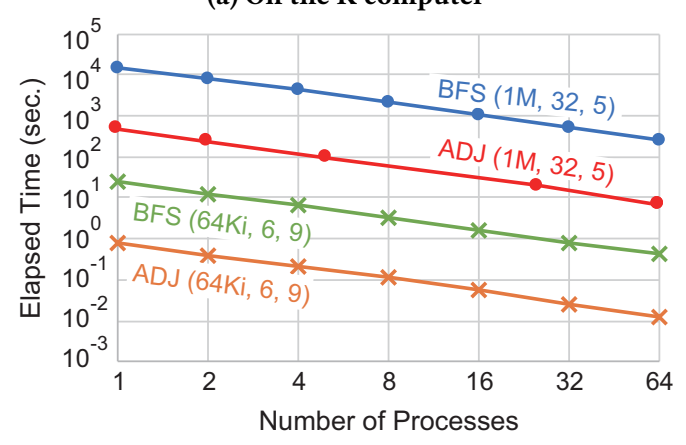

(b) On the Cygnus system

Figure 12: Results of Parallel APSP Algorithm

thread. This result indicates that the parallelization efficiency of BFS-APSP is lower than ADJ-APSP. This is because BFS-APSP requires exclusive control between threads, whereas ADJ-APSP does not perform such a control.

4.3.2 Parallel algorithm by MPI and OpenMP. In this evaluation, the number of threads is set to the maximum value for each environment ( 8 for the K computer and 12 for the Cygnus system), and each APSP algorithm is executed by changing the number of processes. One process is assigned to each CPU. The maximum number of processes in $(64 \mathrm{Ki}, 6,9)$ and $(1 \mathrm{M}, 32,5)$ is 65,536 and $1,000,000$ for BFS-APSP and 1,024 and 15,625 for ADJ-APSP, respectively. In $(1 \mathrm{M}, 32,5)$, Divided ADJ-APSP is executed for up to 25 processes, whereas the normal ADJ-APSP is executed after that.

Fig. 12 shows the performance and it indicates that ADJ-APSP is faster than BFS-APSP for the same number of processes. However, BFS-APSP has a larger maximum number of processes, so it can be seen that BFS-APSP may be faster than ADJ-APSP in $(64 \mathrm{Ki}$, 6 , 9) from Fig. 12a. Besides, Fig. 12a shows that the parallelization efficiency of ADJ-APSP gradually decreases after around 256 processes. The reason is that the calculation efficiency for the logical sum decreases because the number of column elements in the adjacency matrix decreases as the number of processes increases.

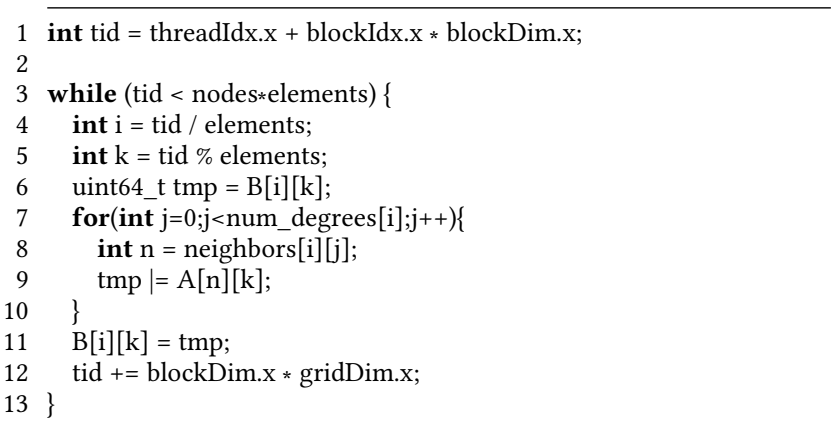

Figure 13: Code of Logical Sum in CUDA

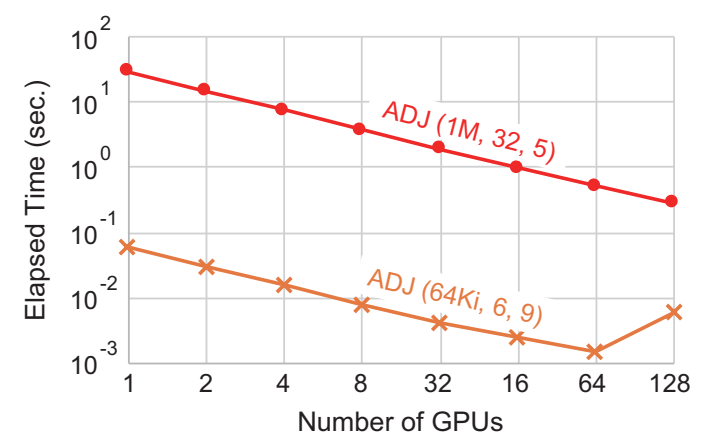

Figure 14: Results of Parallel ADJ-APSP using GPUs

\section{DEVELOPMENT ISSUES}

\subsection{Parallelization using GPU}

The most time-consuming part in ADJ-APSP is the logical sum, which performs continuous memory access. Thus, since its calculation time is limited by the memory bandwidth, it seems to be suitable for accelerators with high-speed memory such as GPUs. As for BFS-APSP, several studies [4, 6] show that GPUs can improve BFS performance to some extent. However, BFS-APSP must be less suitable for GPU than ADJ-APSP because BFS needs an irregular memory access pattern. Thus, this section extends only the parallel ADJ-APSP to execute on GPUs.

Fig. 13 shows the code for the logical sum written in CUDA. To achieve coalesce access, the algorithm is implemented so that sequential threads calculate the logical sum of each row in the adjacency matrix. In the other part, we use the _-popcll() of the CUDA Math API as the function POPCNT() to perform processing on the adjacency matrix on GPU memory. The other details are omitted because their implementations are simple.

We perform a performance evaluation on the Cygnus system in Table 2 which uses graphs of $(n, d, D)=(64 \mathrm{Ki}, 6,9)$ and $(1 \mathrm{M}, 32$, 5 ). Since the compute node has four GPUs and each process uses a single GPU, a maximum of 4 processes are assigned to a single calculation node. We set number of blocks and number of threads as 128 and 1024, respectively. Fig. 14 shows that the elapsed time when using $1 \mathrm{GPU}$ is $6.01 \times 10^{-2}$ seconds for $(64 \mathrm{Ki}, 6,9)$ and 2.87 $\times 10$ seconds for $(1 \mathrm{M}, 32,5)$. These values are 12.47 and 16.53 times 


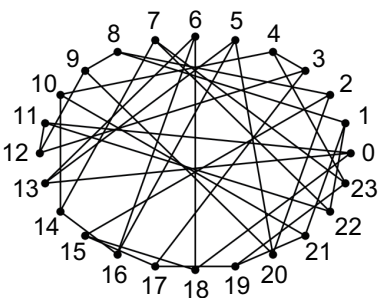

(a) $g=1$

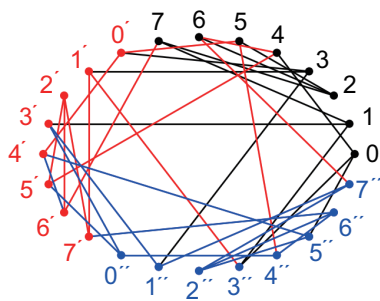

(c) $g=3$

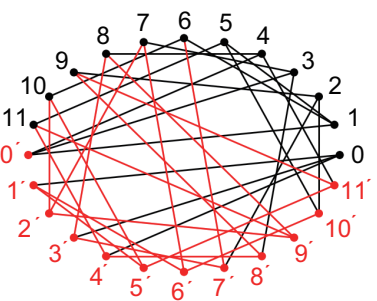

(b) $g=2$

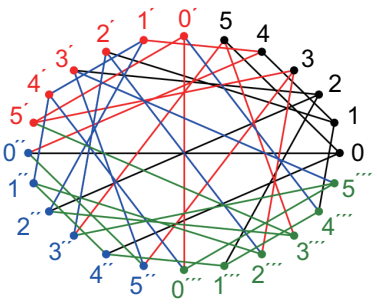

(d) $g=4$
Figure 15: Example of Graph Symmetry $(n, d)=(24,3)[13]$

faster than those in Fig. 10b when using 12 threads of a single CPU. The best value for $(64 \mathrm{Ki}, 6,9)$ using 64 GPUs is $1.59 \times 10^{-3}$ seconds, and that for $(1 \mathrm{M}, 32,5)$ using 128 GPUs is $2.84 \times 10^{-1}$ seconds. We also achieve 38.41- and 101.10-fold performance improvements compared to those when using 1 GPU for $(64 \mathrm{Ki}, 6,9)$ and $(1 \mathrm{M}, 32$, $5)$, respectively. The reason for the performance degradation for $(64 \mathrm{Ki}, 6,9)$ when using 128 processes is that the number of elements of each column in the adjacency matrix is 8 . In this case, the condition where coalesce access occurs (addresses accessed by 16 threads which have adjacent thread numbers) is not met.

\subsection{Application to symmetric input graph}

Our previous research[13] indicates that the computational complexity of BFS-APSP is reduced and the performance of metaheuristic algorithms in the order/degree problem is improved when using the graph symmetry. The research proposed a method for optimization while maintaining symmetry in the graph. Fig. 15 shows examples of graphs with symmetry $(n, d)=(24,3)$ for different numbers of groups, $g$. When a graph is viewed as a plane, if it is rotated by $(360 / g)$ degrees, the connection relationship between edges and the vertices remains the same. Therefore, $g$ must be a divisor of $n$. Note that when $g=1$, it is a normal graph without symmetry. The method for giving symmetric to a graph is as follows. (1) Create a base graph with a number of vertices $(n / g)$ and degree $d$. (2) Duplicate the base graph $g$ times. (3) Select an appropriate edge from each base graph and connect it to the other graphs. Here, it is necessary to satisfy $(n / g)>d$ due to the constraints of the base graph. For more details, refer to [13].

By making the graphs symmetric, the computational complexity of BFS-APSP can be reduced dynamically. The graph has the property that vertices with symmetrical relationships have the same distance between sets of vertices. For example, in Fig. 15d, since

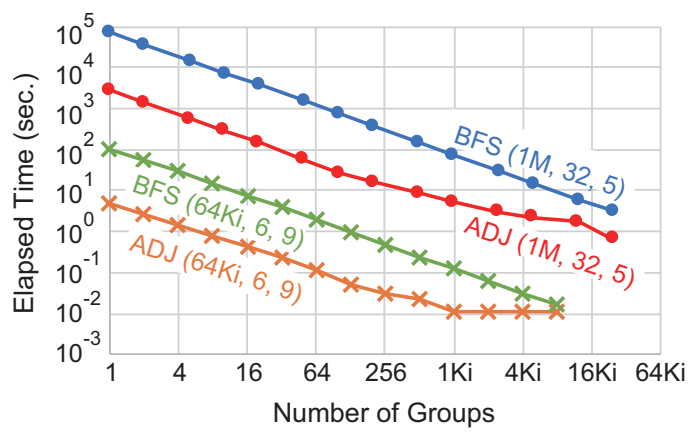

(a) On the K computer

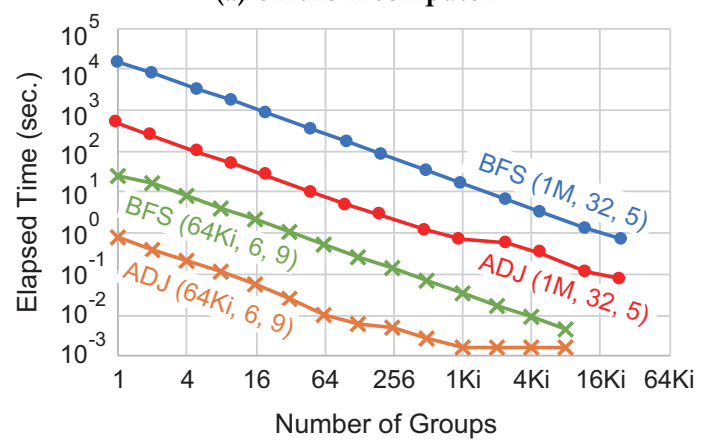

(b) On the Cygnus system

Figure 16: Results for Graph Symmetry on a Single CPU

the set of distances from vertices $0,0^{\prime}, 0^{\prime \prime}, 0^{\prime \prime \prime}$ to the other vertices are all the same, APSP can be obtained by performing BFS only for vertices 0 to 5 . In other words, to obtain APSP using BFSAPSP in a graph with $g$ groups, it is only necessary to perform BFS from $(n / g)$ vertices instead of $n$. As with BFS-APSP, ADJ-APSP can reduce the computational complexity by targeting only $(n / g)$ vertices. The computational complexities of BFS-APSP and ADJAPSP in a graph with $g$ groups are $O\left(n^{2} d / g\right)$ and $O\left(n^{2} d D / g E\right)$, respectively. Note that the maximum number of processes also decreases because the number of vertices processed decreases. The maximum numbers of processes of BFS-APSP and ADJ-APSP are $(n / g)$ and $\lceil(n / g) / E\rceil$, respectively. The value of $\lceil(n / g) / E\rceil$ is the number of elements of the column in the adjacency matrix.

We evaluate the performance of each APSP algorithm using graphs with symmetry. The input graph is assumed to have symmetry. The graphs are $(n, d, D)=(64 \mathrm{Ki}, 6,9)$ and $(1 \mathrm{M}, 32,5)$ created by the procedure outlined in the first paragraph of this section. Because these graphs were optimized using our metaheuristic algorithm[13], the value of diameter $D$ is the same as for the graphs used in the previous section. In the experiment, as in section 4.3.2, the number of threads is set to the maximum value for each computer environment. The number of processes is fixed at 1 , and only the number of groups $g$ is changed. The maximum value of $g$ is 8,192 for $(64 \mathrm{Ki}$, $6,9)$ and 25,000 for $(1 \mathrm{M}, 32,5)$.

Fig. 16 shows the performance and it indicates that ADJ-APSP is faster than BFS-APSP for all cases. However, it can be seen that the speed improvement rate of ADJ-APSP is lower than BFS-APSP as the number of groups $g$ increases. The reason is the same as in 


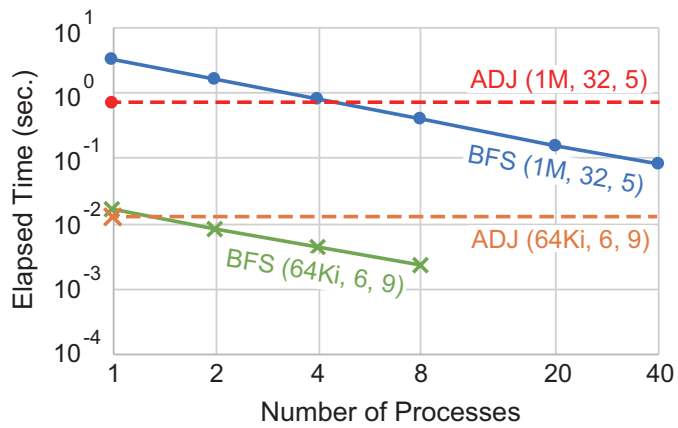

(a) On the K computer

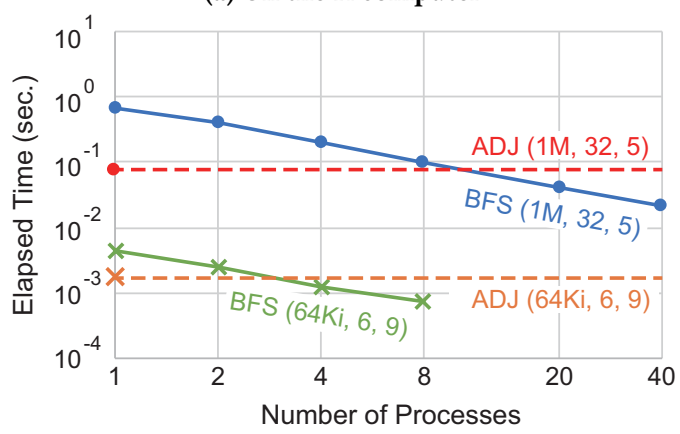

(b) On the Cygnus system

Figure 17: Results for Graph Symmetry on Multiple CPUs

section 4.3.2, because the number of elements for a column in the adjacency matrix decreases as the number of groups $g$ increases. In addition, the elapsed time of ADJ-APSP for $(64 \mathrm{Ki}, 6,9)$ after $g=$ 1,024 does not change. The reason is that the number of elements for a column in the adjacency matrix used in $(64 \mathrm{Ki}, 6,9)$ when $g=1,024$ is 1 , and the computational cost of ADJ-APSP does not change after $g=1,024$.

Next, Fig. 17 shows the results for changing the number of processes while fixing the number of threads and the number of groups to the maximum values. The maximum numbers of processes for $(64 \mathrm{Ki}, 6,9)$ and $(1 \mathrm{M}, 32,5)$ are 8 and 40 for BFS-APSP and are both 1 for ADJ-APSP. Therefore, Fig. 17 uses dotted lines for the values for one process of ADJ-APSP. From these results, it can be seen that BFS-APSP is faster than ADJ-APSP in highly parallel computations because BFS-APSP has a larger maximum number of processes.

\section{CONCLUSION AND FUTURE WORK}

In this paper, we parallelize BFS-APSP and ADJ-APSP using MPI and OpenMP for the order/degree problem. From the results of the performance comparison, ADJ-APSP has a better performance in the serial algorithm and threaded algorithm on a single CPU. However, because the maximum number of processes of BFS-APSP is larger than that of ADJ-APSP, the performance of BFS-APSP on multiple CPUs may be higher using MPI. Besides, by parallelizing ADJ-APSP using a single GPU, the performance is improved up to 16.53-fold compared to using a single CPU. In addition, for parallelization using MPI and multiple GPUs, we also achieve up to 101.10-fold times performance improvement compared to using a single GPU. This paper also indicates that the computational complexities of BFS-APSP and ADJ-APSP can be greatly reduced by giving symmetry to graphs. However, since the maximum number of processes of ADJ-APSP is decreased further in the case using symmetry, the performance of BFS-APSP may exceed that of ADJAPSP with a relatively small number of processes.

The following are examples of topics for future work. (1) Further parallelize each APSP algorithm. In the parallelization of BFS-APSP in this paper, only thread parallelization is used for a single BFS, but MPI parallelization is also possible for a single BFS[3]. Also, ADJ-APSP can be further parallelized by dividing the adjacency matrix horizontally. (2) In order to improve convenience for users, develop a function to automatically select a high-speed APSP algorithm according to an input graph and a computer environment. To develop such functionality, it is necessary to model each APSP algorithm.

\section{ACKNOWLEDGEMENTS}

This research used the Cygnus system provided by Interdisciplinary Computational Science Program in the Center for Computational Sciences, University of Tsukuba. This work was supported by JSPS KAKENHI Grant Number 18K11331 and RIKEN Incentive Research Projects. We express our sincere thanks to Dr. Ryuhei Mori for an excellent implementation of the serial version ADJ-APSP and Divided ADJ-APSP. We are grateful to Dr. Yutaka Maruyama and Dr. Daichi Mukunoki for helpful discussions about the GPU version of ADJ-APSP, and also grateful to the Graph Golf committee for giving us the interesting issues.

\section{REFERENCES}

[1] [n.d.]. The Degree/Diameter Problem - Combinatorics Wiki. http://combinatoricswiki.org/wiki/The_Degree/Diameter_Problem.

[2] Ankit Singla et al. 2011. Jellyfish: Networking Data Centers Randomly. CoRR abs/1110.1687 (2011). arXiv:1110.1687 http://arxiv.org/abs/1110.1687

[3] Andy Yoo et al. 2005. A Scalable Distributed Parallel Breadth-First Search Algorithm on BlueGene/L. In SC '05: Proceedings of the 2005 ACM/IEEE Conference on Supercomputing. 25-25. https://doi.org/10.1109/SC.2005.4

[4] Hui Guo et al. 2018. Accelerating BFS via Data StructureAware Prefetching on GPU. IEEE Access 6 (2018), 60234-60248. https://doi.org/10.1109/ACCESS.2018.2876201

[5] Jurij Leskovec et al. 2005. Realistic, Mathematically Tractable Graph Generation and Evolution, Using Kronecker Multiplication. In Knowledge Discovery in Databases: PKDD 2005. Springer Berlin Heidelberg, Berlin, Heidelberg, 133-145.

[6] Lijuan Luo et al. 2010. An Effective GPU Implementation of Breadth-first Search. In Proceedings of the 47th Design Automation Conference (DAC '10). 5255. https://doi.org/10.1145/1837274.1837289

[7] Nicholas Metropolis et al. 1953. Equation of State Calculations by Fast Computing Machines. The Journal of Chemical Physics 21, 6 (1953), 1087-1092.

[8] Scott Beamer et al. 2012. Direction-optimizing Breadth-first Search. In Proceedings of the International Conference on High Performance Computing, Networking, Storage and Analysis (SC '12). IEEE Computer Society Press, Los Alamitos, CA, USA, Article 12, 10 pages.

[9] Scott Kirkpatrick et al. 1983. Optimization by Simulated Annealing. Science 220, 4598 (1983), 671-680.

[10] Virat Agarwal et al. 2010. Scalable Graph Exploration on Multicore Processors. In Proceedings of the 2010 ACM/IEEE International Conference for High Performance Computing, Networking, Storage and Analysis (SC '10). IEEE Computer Society, Washington, DC, USA, 1-11. https://doi.org/10.1109/SC.2010.46

[11] Hiroki Matsutani et al. 2014. Low-latency wireless 3D NoCs via randomized shortcut chips. In 2014 Design, Automation Test in Europe Conference Exhibition (DATE). 1-6. https://doi.org/10.7873/DATE.2014.286

[12] Ji-Yong Shin et al. 2011. Small-world Datacenters. In Proceedings of the 2Nd ACM Symposium on Cloud Computing (SOCC '11). ACM, New York, NY, USA, Article 2, 13 pages. https://doi.org/10.1145/2038916.2038918 
[13] Masahiro Nakao et al. 2019. A Method for Order/Degree Problem Based on Graph Symmetry and Simulated Annealing with MPI/OpenMP Parallelization. In Proceedings of the International Conference on High Performance Computing in Asia-Pacific Region (HPC Asia 2019). ACM, 128-137. https://doi.org/10.1145/3293320.3293325

[14] Michihiro Koibuchi et al. 2012. A case for random shortcut topologies for HPC interconnects. In 2012 39th Annual International Symposium on Computer Architecture (ISCA). 177-188. https://doi.org/10.1109/ISCA.2012.6237016

[15] Michihiro Koibuchi et al. 2016. Optical network technologies for HPC: computer-architects point of view. IEICE Electronics Express 13, 6 (2016), 1-14 https://doi.org/10.1587/elex.13.20152007

[16] Michihiro Koibuchi et al. 2018. Graph Golf: The Order/degree Problem Competition. http://research.nii.ac.jp/graphgolf.

[17] Nobutaka Shimizu and Ryuhei Mori. 2016. Average Shortest Path Length of Graphs of Diameter 3. CoRR abs/1606.05119 (2016). arXiv:1606.05119 http://arxiv.org/abs/1606.05119

[18] Robert W. Floyd. 1962. Algorithm 97: Shortest Path. Commun. ACM 5, 6 (June 1962), 345. https://doi.org/10.1145/367766.368168
[19] Ryosuke Mizuno and Yawara Ishida. 2016. Constructing large-scale low-latency network from small optimal networks. In 2016 Tenth IEEE/ACM International Symposium on Networks-on-Chip (NOCS). 1-5. https://doi.org/10.1109/NOCS.2016.7579336

[20] Ryuhei Mori. [n.d.]. https://github.com/ryuhei-mori/graph_ASPL.

[21] Raimund Seidel. 1995. On the All-Pairs-Shortest-Path Problem in Unweighted Undirected Graphs. Fournal of Computer and System Sciences 51, 3 (1995), 400403. https://doi.org/10.1006/jcss.1995.1078

[22] Stephen Warshall. 1962. A Theorem on Boolean Matrices. F. ACM 9, 1 (Jan 1962), 11-12. https://doi.org/10.1145/321105.321107

[23] Takafumi Watanabe et al. 2008. Impact of topology and link aggregation on PC cluster with Ethernet. In 2008 IEEE International Conference on Cluster Computing. 280-285. https://doi.org/10.1109/CLUSTR.2008.4663782

[24] Teruaki Kitasuka and Masahiro Iida. 2016. A Heuristic Method of Generating Diameter 3 Graphs for Order/Degree Problem. CoRR abs/1609.03136 (2016). arXiv:1609.03136 http://arxiv.org/abs/1609.03136 